\title{
Extended Cranial Ultrasound Views in Infants with Acute Brain Stem/Infratentorial Lesions: Diagnosis of a Progressive Midline Glioma in a 6-Week-Old Infant
}

\author{
Matthias Lange ${ }^{1}$ Bernd Mitzlaff ${ }^{1}$ Florian Beske ${ }^{1}$ Holger Koester $^{1}$ Wiebke Aumann $^{2}$ \\ Johannes Woitzik ${ }^{2}$ Hermann L. Mueller ${ }^{1}$ Axel Heep ${ }^{1}$
}

${ }^{1}$ Department of Neonatology, Pediatric Intensive Care and Cardiology, University of Oldenburg, Oldenburg, Germany

2 Department of Neurosurgery, Evangelisches Krankenhaus Oldenburg, Oldenburg, Germany

J Child Sci 2021;11:e262-e264.

Address for correspondence Matthias Lange, MD, Department of Neonatology, Pediatric Intensive Care and Cardiology, University of Oldenburg, Rahel-Straus-Straße 10, Oldenburg 26133, Germany (e-mail: Lange.Matthias@klinikum-oldenburg.de).

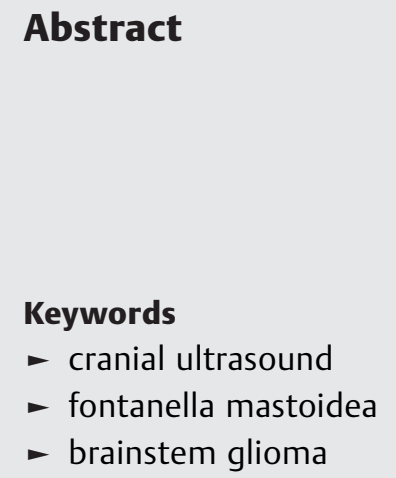

Central nervous system (CNS) tumors are the most common solid tumors in children and adolescents. However, in neonates and children aged younger than a year, they are very rare. Clinical presentation in neonates is often subtle and nonspecific. When neurological symptoms are apparent at this age, cranial ultrasound (CUS) is often done as the initial evaluation, with a standard approach through the anterior fontanel (AF), followed by further imaging, such as magnetic resonance imaging (MRI), if necessary. We report the first neonatal case of a rapidly progressive diffuse midline glioma positive for histone H3 K27M mutation (World Health Organization [WHO] grade IV) in which using extended (transmastoid) CUS studies through the mastoid fontanelle (MF) in the second month of life defined the lesion in the brainstem.

\section{Introduction}

Transcranial ultrasonography is a reliable, inexpensive, and noninvasive tool and therefore often used for initial bedside diagnostics when neurological or nonspecific symptoms (e.g., seizure, elevated anterior fontanel [AF], and lethargy) are apparent in neonates and infants in the first months of life. The standard cranial ultrasound (CUS) approach is through the $\mathrm{AF}$ in sagittal and coronal projections. The standard transducer frequency for neonatal CUS is 8 to $11 \mathrm{MHz}$. A better depth penetration can be reached by decreasing the transducer frequency $(5-6 \mathrm{MHz})$. However, lower frequencies reduce the object resolution of the ultrasound image. ${ }^{1}$

For the depiction of the posterior fossa (PF) in preterm infants and neonates, an extremely small high-frequency microconvex transducer is used $(8-11 \mathrm{MHz}$ with the standard microconvex probes and even higher, up to $15-18 \mathrm{MHz}$, with linear probes). The mastoid fontanelles (MFs) are located at the

received

December 25, 2020

accepted after revision

August 8, 2021 intersection of the parietal, temporal, and occipital bones. ${ }^{2}$ To perform the examination using the MF as an acoustic window, the examiner places the transducer in the mastoid area behind the ear and then moves and changes direction until able to view the PF. Images should be documented in axial (also transverse) and coronal planes at differential levels. ${ }^{1,3}$ - Figs. $\mathbf{1 A}$ and $\mathbf{1 B}$ show the anatomic structures of a neonatal skull and the axial position of the transducer. Starting the examination with the probe caudally angled allows visualization of the cisterna magna, cerebellar vermis, fourth ventricle, and both cerebellar hemispheres. Sweeping the probe in the rostral direction allows for visualization of the tentorium, aqueduct, and pedunculi. The posterior horn of the lateral ventricle and the associated choroid plexus can be detected in the supratentorial area. Near the transducer, the flow of the transverse and sigmoid sinuses can be shown which can be useful in the diagnosis of sinus vein thrombosis. ${ }^{4}$-Fig. $\mathbf{2}$ is a coronal view through the MF showing the normal anatomy of the PF. The neonate may

\section{(c) 2021. The Author(s)}

This is an open access article published by Thieme under the terms of the Creative Commons Attribution License, permitting unrestricted use, distribution, and reproduction so long as the original work is properly cited. (https://creativecommons.org/licenses/by/4.0/) Georg Thieme Verlag KG, Rüdigerstraße 14, 70469 Stuttgart, Germany 


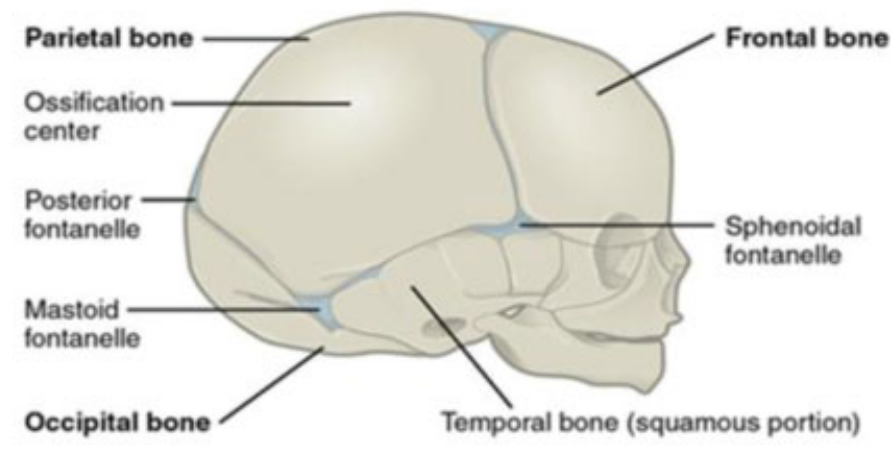

A

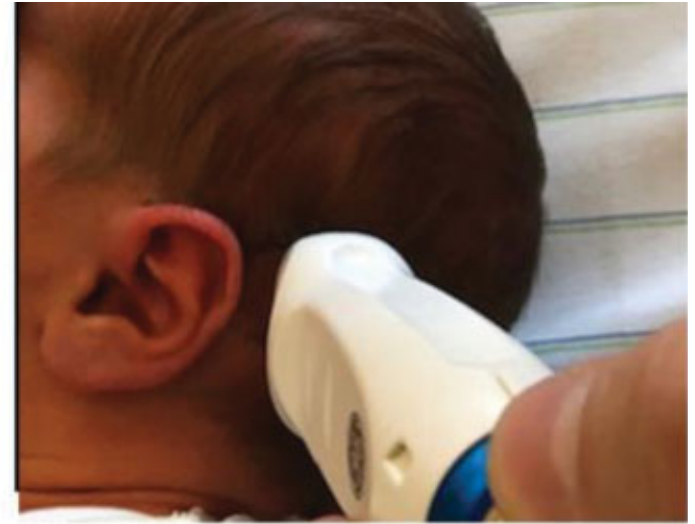

$\mathbf{B}$

Fig. 1 (A) Anatomic structure of a newborn scull (http://radiopedia.org/images/19305134); (B) retroauricular position of the convex probe.

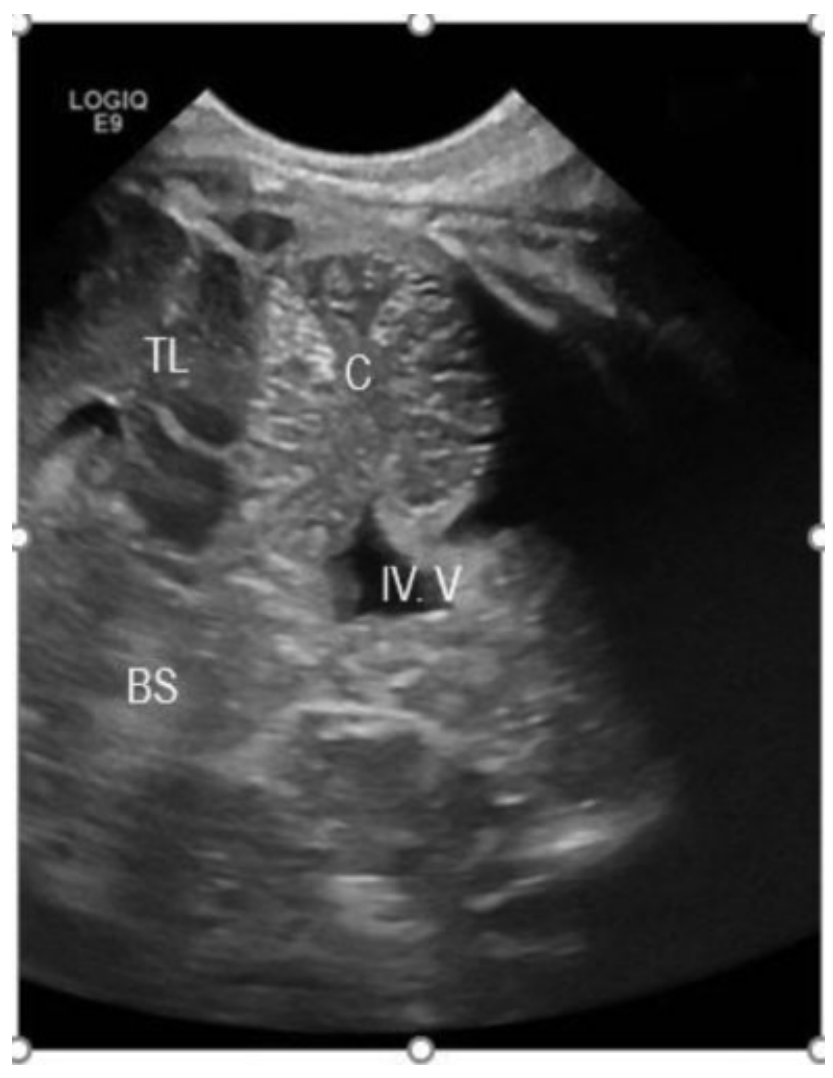

Fig. 2 Coronary cranial ultrasound through the mastoid fontanelle (MF) of a healthy newborn. Cerebellum (C), fourth ventricle (IV, V), Cisterna magna, temporal lobe (TL), brainstem (BS).

experience an auditory response to pulses of radiofrequency energy which could cause discomfort. ${ }^{5}$ For this reason, the views through the MFs should be performed at the end of the examination.

\section{Case Presentation}

Following an uneventful pregnancy, a 5-week-old girl presented with moderate inspiratory stridor at a local medical facility. On clinical examination, a benign neonatal laryngomalacia was suspected. However, due to the progression of symptoms, she was admitted to our hospital. On admission, she showed signs of severe inspiratory stridor but otherwise uneventful clinical and neurological examination. Echocardiography excluded aortic anomalies, such as a vascular ring. Elective bronchoscopy demonstrated dysfunctional inspiratory motility of vocal cords but no signs of tracheomalacia. During the first 3 days of admission, the patient's clinical condition deteriorated, and the infant developed neurological symptoms including nystagmus, muscular hypotonia, and apnoeic episodes. On admission to the pediatric intensive care unit, the clinical examination revealed a bulging fontanelle, muscular hypotonia, and lethargy.

Immediately, point of care ultrasound was performed and showed a heteroechogenic lesion in the brain stem (-Fig. 3A). Subsequently, magnetic resonance imaging (MRI; - Fig. 3B) confirmed the infratentorial tumor extension localized in the brainstem. The patient underwent emergency tumor biopsy and external ventricular drain. The histopathological results confirmed a diffuse midline glioma, histone H3 K27M mutation, which has a poor prognosis. Sadly, despite external ventricular drainage, neurological symptoms of obstructive hydrocephalus progressed, and following review of therapeutic options, the patient was placed on palliative care at the age of 7 weeks. Standard CUS window via the AF demonstrated enlarged lateral ventricles but was unable to visualize the infratentorial structures. Additional CUS views via the MF were obtained which showed a $2.5 \times 1.8 \mathrm{~cm}$ heteroechogenic lesion with poorly differentiated borders. The texture of the mass seemed to be nonhomogeneous, with isolated cystic structures within it. There was no increased vascularity, and the mass did not appear to invade the surrounding tissue. All in all, the mass was suspected to be a brain stem glioma with secondary obstruction of the third ventricle (-Fig. $\mathbf{3 A}$ and $\mathbf{3 B}$ ).

\section{Discussion}

Brain tumors in neonates and children younger than a year of age are very rare, and their clinical presentation is often nonspecific and subtle. ${ }^{6}$

Satrom et al reported in 2017 about a newborn with respiratory failure caused by a congenital diffuse 

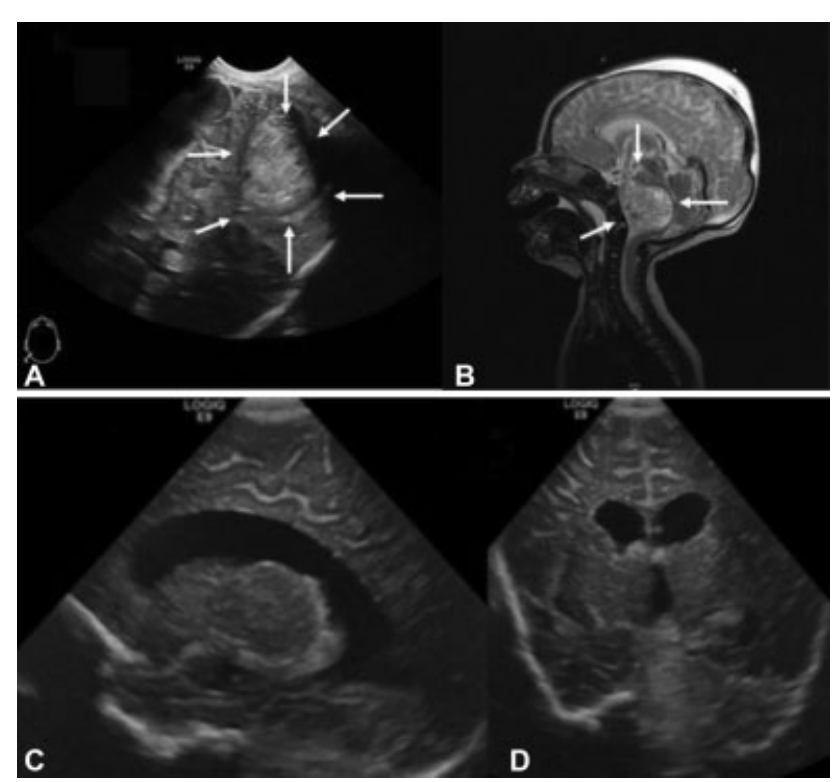

Fig. 3 (A) Transcranial ultrasound through the MF showing a heterogenic mass in the PF, localized in the brainstem. (B) T1weighted images shows inhomogeneous mass with displacement of fourth ventricle (arrows show the border of the tumor). (C and D) Transcranial ultrasound through the anterior fontanel (AF), axial and coronal view.

intrinsic pontine glioma. The tumor was only detected by MRI scan. ${ }^{7}$

Diffuse midline gliomas with $\mathrm{H} 3 \mathrm{~K} 27 \mathrm{M}$ mutation have a dismal prognosis regardless of their location, the histopathological grading, or the extent of tumor resection. The median survival of these gliomas is less than 1 year. ${ }^{8}$

CUS is the primary neonatal neuroimaging technique. It can be performed early and repeatedly at the bedside and provides instant diagnostic information. The standard CUS view is obtained through the AF. This enables reliable views of supratentorial structures, but visualization of the PF is less optimal due to its distance away from the tranducer. ${ }^{9}$ The standard views ( - Fig. 3C and 3D) are inferior to the view through the MF since the echogenic tentorium and vermis may impede visualization. Also, pathological findings, such as the extension of the lateral ventricle, can complicate the evaluation of the PF. The MF enables a better view of the PF. ${ }^{10,11}$

A study from 2015 compared the evaluation of the PF using CUS through the AF and in addition to the PF. The pathological findings were compared with MRI findings. The study showed that the additional examination via the MF increased the sensitivity, as well as the specificity, of the cranial ultrasound. ${ }^{12}$ Recently, the same working group published a detailed description of the examination of the fossa posterior. ${ }^{13}$ Specifically, in preterm infants, CUS has reached a new significance due to the possibility of detecting the smallest bleedings in the cerebellum. CUS examination for hypoxic-ischemic encephalopathy in term infants should include views through the MF. Furthermore, the CUS via the MF can be useful when suspecting an intracerebral infection or malformation, such as Arnold Chiari and Dandy Walker. To our knowledge, there is no report of a diffuse midline glioma that has been detected via ultrasound through the MF.

\section{Conclusion}

Our case report demonstrated the benefit of using ultrasound view through the MF. This extended ultrasound window is most valuable for evaluating PF malformations as it provides highly detailed anatomic views of the cerebellum, fourth ventricle, and cisterna magna. In the presented case, the lesion and secondary CSF flow obstruction were detected on bedside examination, guiding further diagnostic modalities (MRI), as well as indication for release of ventricular dilatation in occlusive HC.

Conflict of Interest

None declared.

\section{References}

1 Steggerda SJ, van Wezel-Meijler G. Cranial ultrasonography of the immature cerebellum: Role and limitations. Semin Fetal Neonatal Med 2016;21(05):295-304

2 Meijler G, Steggerda SJ. Neonatal Cranial Ultrasonography. 3rd ed. Switzerland: Springer, Nature; 2019

3 Enriquez G, Correa F, Aso C, et al. Mastoid fontanelle approach for sonographic imaging of the neonatal brain. Pediatr Radiol 2006; 36(06):532-540

4 Raets MMA, Sol JJ, Govaert P, et al. Serial cranial US for detection of cerebral sinovenous thrombosis in preterm infants. Radiology 2013;269(03):879-886

5 Elder JA, Chou CK. Auditory response to pulseradiofrequency energy. Bioelectromagnetics 2003;6:1274-1282

6 Louis DN, Perry A, Reifenberger G, et al. The 2016 World Health Organization classification of tumors of the central nervous system: a summary. Acta Neuropathol 2016;131(06):803-820

7 Satrom KM, Phelan RA, Moertel CL, Brent Clark H, Johnson DE, George TN. Neonatal respiratory failure caused by congenital diffuse intrinsic pontine glioma. J Child Neurol 2017;32(06): 533-536

8 Karremann M, Gielen GH, Hoffmann M, et al. Diffuse high-grade gliomas with $\mathrm{H} 3 \mathrm{~K} 27 \mathrm{M}$ mutations carry a dismal prognosis independent of tumor location. Neuro-oncol 2018;20(01):123-131

9 Steggerda SJ, Leijser LM, Walther FJ, van Wezel-Meijler G. Neonatal cranial ultrasonography: how to optimize its performance. Early Hum Dev 2009;85(02):93-99

10 Teele RL, Taylor GA. Demonstration of fourth ventricular choroid plexus on neonatal cranial ultrasonography. Pediatr Radiol 2012; 42(05):620-623

11 Di Salvo DN. A new view of the neonatal brain: clinical utility of supplemental neurologic US imaging windows. Radiographics 2001;21(04):943-955

12 Steggerda SJ, de Bruïne FT, Smits-Wintjens VE, Verbon P, Walther FJ, van Wezel-Meijler G. Posterior fossa abnormalities in high-risk term infants: comparison of ultrasound and MRI. Eur Radiol 2015; 25(09):2575-2583

13 Fumagalli M, Parodi A, Ramenghi L, Limperopoulos C, Steggerda SeurUS brain group. Ultrasound of acquired posterior fossa abnormalities in the newborn. Pediatr Res 2020;87 (Suppl 1):25-36 